incubated for $20 \mathrm{~h}$ following which the migrated cells were fixed, stained and counted.

Results HSCs demonstrated greater migration towards medium obtained from HSCs cultured for 3 days under hypoxic conditions compared to normoxic HSCs $(p=0.06)$. This effect decreased with longer culture times, reaching levels significantly lower than baseline by day 7 for both groups $(p<0.0001)$. In contrast, medium obtained from HSCs cultured on gel matrix under normoxic conditions stimulated significantly higher HSC migration compared to hypoxic qHSCs, with a peak effect by day $5(\mathrm{p}<0.0001)$. Similarly, normoxic KCs stimulated significantly enhanced HSCs migration compared to hypoxic KCs $(p<0.0001)$. On the other hand, hypoxic BECs attracted significantly more HSCs on day 1 compared to normoxia, an effect that continued to rise on day $7(p=0.002)$.

Conclusion This study demonstrates that hypoxia in an in vitro model stimulates contrasting responses depending on cell type and activation state. KCs and qHSCs in a relatively high $\mathrm{O} 2$ state similar to that of reperfusion during liver surgery can promote chemotaxis of aHSCs possibly through the formation of reactive oxygen species. BECs and aHSCs appear to produce factors that negatively affect HSC chemotaxis as evident by below-baseline migration responses. Hypoxia attenuates this negative effect in vitro.

Competing interests None declared.

\section{PTU-054 TRANSJUGULAR INTRAHEPATIC PORTOSYSTEMIC SHUNTS FOLLOWING LIVER TRANSPLANTATION CAN BE ASSOCIATED WITH A GOOD PROGNOSIS: A SINGLE CENTRE EXPERIENCE}

doi:10.1136/gutjnl-2012-302514c.54

${ }^{1} B$ Hogan, * ${ }^{1} \mathrm{D}$ Thorburn, 'D Sharma, ${ }^{1} \mathrm{~B}$ Davidson, ${ }^{1} \mathrm{~J}$ P O'Beirne, 'A K Burroughs, ${ }^{2} \mathrm{D}$ Yu, ${ }^{1} \mathrm{D}$ W Patch. ${ }^{1}$ Sheila Sherlock Liver Centre, Royal Free Hospital, London, UK; ${ }^{2}$ Department of Interventional Radiology, Royal Free Hospital, London, UK

Introduction Transjugular intrahepatic portosystemic shunt (TIPS) is indicated in the management of portal vein thrombosis or stenosis, portal hypertension and for veno-occlusive disease in post-liver transplant (LT) patients. Previous series have reported 1-year mortality rates of $14 \%-67 \%$. A MELD score $>15$ at the time of insertion may indicate a poor long term prognosis. We aimed to evaluate the safety of TIPS after LT at a UK tertiary referral centre.

Methods We retrospectively analysed data from the Royal Free Hospital TIPS database between 1st January 1991 and the 31st January 2011. All patients who had undergone TIPS following LT were included.

Results During the period studied 629 patients received a TIPS. In the same period 1192 liver transplant operations were performed. 10 TIPS were inserted into patients following LT for recurrent cirrhosis with refractory ascites (4), veno-occlusive disease (3) and portal vein thrombosis (3). The original indications for transplantation were PSC (3), PBC (3), Hepatitis C (1), Autoimmune (1), Primary Oxalosis (1) and Acute Liver Failure (1). We noted a median survival of 38 months. Survival at 1 and 5 years was $100 \%$ and $60 \%$ respectively. The median MELD at the time of TIPS insertion was 12 (range 7-19). No correlation between the MELD score at the time of TIPS insertion and survival was demonstrated $(p=0.62)$.

Conclusion These results suggest that TIPS can be performed safely after LT and that survival rates better than those previously reported can be achieved. We suggest TIPS should be used in carefully selected candidates following LT as a definitive treatment for patients not suitable for re-transplant or as a bridge to re-transplantation. The alternative of re-transplantation should always be considered prior to TIPS insertion where indicated.

\section{Competing interests None declared.}

\section{REFERENCES}

1. Saad WEA, et al. Transjugular intrahepatic portosystemic shunts in liver transplant recipients for management of refractory ascites: clinical outcome. J Vasc Interv Radiol 2010;21:218-23.

2. Kim JJ, et al. Transjugular intrahepatic portosystemic shunts in liver transplant recipients. Liver Int 2008;28:240-8.

3. Feyssa $\mathbf{E}$, et al. MELD score less than 15 predicts prolonged survival after transjugular intrahepatic portosystemic shunt for refractory ascites after liver transplantation. Transplantation 2011;91:786-92.

\section{PTU-055 THE ASSOCIATION OF CO-EXISTENT NON-ISCHAEMIC CARDIAC DISEASE WITH MORBIDITY AND MORTALITY FOLLOWING LIVER TRANSPLANTATION}

doi:10.1136/gutjnl-2012-302514c.55

${ }^{1} \mathrm{H}$ Steed, ${ }^{*}{ }^{1} \mathrm{~J}$ A Leithead, ${ }^{1} \mathrm{~K}$ Kandiah, ${ }^{2} \mathrm{~B}$ K Gunson, ${ }^{1} \mathrm{~J}$ W Ferguson. ${ }^{1}$ Liver Unit, Queen Elizabeth Hospital, Birmingham, UK; ${ }^{2}$ NIHR Biomedical Research Unit and Center for Research, University of Birmingham, Birmingham, UK

Introduction The risk stratification of potential liver transplant candidates with co-existent non-ischaemic cardiac disease (CD) is particularly challenging given the masking effect of the haemodynamic dysfunction of advanced portal hypertension. Whether such patients have altered outcomes following liver transplantation remains unknown. Our aim was, therefore, to examine the effects of mild and moderate non-ischaemic $\mathrm{CD}$ on post liver transplant morbidity and mortality.

Methods Single-centre study of patients undergoing first liver transplantation for chronic liver disease. To determine the effects of mild $\mathrm{CD}$, the routine assessment trans-thoracic echocardiography reports of 246 patients transplanted 01/2007-03/2010 were reviewed. To determine the effects of moderate $\mathrm{CD}$, patients transplanted from 1994-present were highlighted from a prospectively collected database.

Results Mild CD $11.4 \%, 11.4 \%, 2.8 \%$, and $0.8 \%$ of the 246 patients undergoing routine assessment echocardiography had left atrial dilatation, or mild mitral regurgitation, aortic regurgitation or aortic stenosis, respectively. After adjusting for confounding variables there was no association between left atrial dilatation $(p=0.738)$ or mild mitral regurgitation $(p=0.234)$ and a cardiac event post transplant. Similarly, there was no association between left atrial dilatation $(p=0.146)$ or mild mitral regurgitation $(p=0.157)$ and post transplant survival. Moderate CD Ten patients were transplanted with moderate $\mathrm{CD}$ : aortic valve replacement (4), moderate valvular dysfunction (2), HOCM (2), moderate left ventricular failure (1), mitral valvuloplasty (1). Two of these patients underwent simultaneous valve replacement surgery. Of these two patients, one had a peri-operative cardiac event and both were alive at 1-year. For the remaining eight patients who did not undergo intervention the cumulative incidence of a cardiac event by 1-year after transplantation was $68.7 \%$, and the estimated 1 -year survival was $46.7 \%$. All three patients with a pre-existing aortic valve replacement that was not re-done had a cardiac complication: one patient developed bacterial endocarditis and awaits further valve replacement surgery, two died of cardiac failure at 90- and 200-days post transplant.

Conclusion Chronic liver disease patients with co-existent moderate $\mathrm{CD}$ who receive a favourable cardiac risk assessment have greater than expected morbidity and mortality following liver transplantation. Our findings suggest that the severity of non-ischaemic $\mathrm{CD}$ is underestimated in this setting.

Competing interests None declared. 